OPEN ACCESS

Edited by:

Javier Moreno,

Carlos III Health Institute, Spain

Reviewed by:

Joseli Lannes-Vieira

Oswaldo Cruz Foundation

(Fiocruz), Brazil

Paola Lasso,

Pontifical Javeriana

University, Colombia

*Correspondence:

Miriam Postan

miriampostan@yahoo.com

Specialty section:

This article was submitted to

Parasite and Host,

a section of the journal

Frontiers in Cellular and Infection

Microbiology

Received: 21 October 2019 Accepted: 06 March 2020

Published: 31 March 2020

Citation:

Quebrada Palacio LP, Fernández ER, Hernández-Vásquez Y, Petray PB and

Postan M (2020) Circulating T

Follicular Helper Cell Abnormalities Associated to Different Clinical Forms

of Chronic Chagas Disease.

Front. Cell. Infect. Microbiol. 10:126.

doi: $10.3389 / f c i m b .2020 .00126$

\section{Circulating T Follicular Helper Cell Abnormalities Associated to Different Clinical Forms of Chronic Chagas Disease}

\author{
Luz P. Quebrada Palacio ', Esteban R. Fernández', Yolanda Hernández-Vásquez ${ }^{1}$, \\ Patricia B. Petray ${ }^{2,3}$ and Miriam Postan ${ }^{1,3 *}$ \\ ${ }^{1}$ Instituto Nacional de Parasitología "Dr. Mario Fatala Chabén", ANLIS/Malbran, Buenos Aires, Argentina, ${ }^{2}$ Facultad de \\ Medicina, IMPaM-Instituto de Investigaciones en Microbiología y Parasitología Médica (UBA-CONICET), Buenos Aires, \\ Argentina, ${ }^{3}$ Consejo Nacional de Investigaciones Cientificas y Tecnológicas (CONICET), Buenos Aires, Argentina
}

Multiple perturbations of the immune response affecting a range of cells have been reported in Trypanosoma cruzi-infected individuals and associated to clinical manifestations of chronic Chagas disease. There is a paucity of knowledge about the role of $T$ follicular helper (Tfh) cells in this infection. Here, we sought to characterize circulating Tfh (cTfh) cells in chronic Chagas disease patients and to identify potential associations with disease severity in humans. cTfh cells were characterized by flow cytometry in freshly isolated PBMCs from $7 \mathrm{~T}$. cruzi-infected asymptomatic patients (ASYMP), 5 patients with chronic chagasic dilated cardiomyopathy (CCC) and 8 healthy controls, using antibodies against chemokine receptors CXCR5, CXCR3, CCR6, and CCR7. Our results showed significant expansion of CD4+CD45RO+CXCR5+CCR6+ cells in ASYMP and CCC patients, along with a contraction of CD4+CD45RO+CXCR5+CXCR3-CCR6- (cTfh2) cells. ASYMP patients further exhibited decreased CD4+CD45RO+CXCR5+CXCR3+CCR6- (cTfh1) cells and expanded CD4+CD45RO+CXCR5+CXCR3-CCR6+ (cTfh17) cells while CCC patients exhibited significantly increased frequencies of $\mathrm{CD} 4+\mathrm{CD} 45 \mathrm{RO}+\mathrm{CXCR} 5+\mathrm{CCR} 7+$ cells. Linear regression analysis revealed a positive trend of CD4+CD45RO+CXCR5+CXCR3+CCR6+ (cTfh1/17) cells and negative trends of cTfh1 and cTfh2 cells as disease was more severe. There was no correlation between the frequencies of cTfh cells and circulating CD19+lgD-lgG+ cells or serum levels of $T$. cruzi-specific IgG. These results demonstrate that the cTfh compartment of humans chronically infected with T. cruzi comprises expanded CCR6-expressing cells and reduced cTfh2 cells. The association of discrete phenotypic changes in cTfh subsets with different clinical forms suggests the potential contribution of $T$ follicular helper cells to Chagas heart disease progression.

Keywords: T follicular helper cells, circulating Tfh subsets, CXCR3, CCR6, Human Chagas disease, Trypanosoma cruzi 


\section{INTRODUCTION}

Trypanosoma cruzi, the etiological agent of Chagas disease, causes a broad spectrum of clinical outcomes, ranging from a mild, frequently asymptomatic acute illness with low mortality rate to a potentially life-threatening cardiomyopathy, digestive and/or neurological abnormalities in up to $30 \%$ of chronically infected individuals (WHO World Health Organization, 2018). Diverse parasite and host factors have been proposed to account for the differential clinical evolution of Chagas disease (Bryan et al., 2010; Dutra et al., 2014). Trypanosoma cruzi is a heterogeneous species and infection with different strains may result in different clinical outcomes (Quebrada Palacio et al., 2018).

Several parasite proteins have been shown to promote parasite invasion, replication and survival (Watanabe Costa et al., 2016). These molecules might also subvert the complement system and favor intracellular parasite growth, thus hampering the development of parasite-specific innate and adaptive immune responses (Cardoso et al., 2016). Several defects of the $\mathrm{T}$ cell compartment have been associated with the progression of asymptomatic T. cruzi infection to Chagas heart disease. Asymptomatic patients are known to develop balanced Th1/Th2/Th17/Treg adaptive immune responses, with effector cells producing proinflammatory cytokines such as IFN- $\gamma$, TNF$\alpha$ and IL-17, and regulatory cells producing IL-10, while patients with the cardiac form of chronic Chagas disease exhibit a predominantly proinflammatory cytokine profile with limited production of IL-10 (Gomes et al., 2003; Vitelli-Avelar et al., 2008; Dutra et al., 2014). Various B cell defects have also been reported in T. cruzi -infected individuals, including polyclonal B cell activation, hypergammaglobulinemia and secretion of non-specific antibodies and autoantibodies, which have been suggested to delay parasite-specific humoral responses, thus contributing to parasite persistence and pathology (Minoprio et al., 1988; Bryan et al., 2010; Bermejo et al., 2011; Cunha-Neto et al., 2011).

CD4+ $\mathrm{T}$ follicular helper ( $\mathrm{Tfh}$ ) cells are a distinct subpopulation of cytokine-producing $\mathrm{CD} 4+\mathrm{T}$ helper cells that express the chemokine receptor CXCR5 and provide support to cognate B cell responses in germinal centers of secondary lymphoid organs (Breitfeld et al., 2000; Schaerli et al., 2000; Nurieva et al., 2008). A population of CD4+CXCR5+ cells has also been described in the peripheral blood (cTfh) and proposed to represent the circulating counterpart of bona fide Tfh cells. As for Th1, Th2 and Th17 cells (AcostaRodriguez E et al., 2007), the chemokine receptors CXCR3 and CCR6 have been used to define functionally distinct cTfh cell subsets (Morita et al., 2011; Schmitt et al., 2014). In humans, CD4+CD45RO+CXCR5+CXCR3+CCR6(cTfh1) cells are known to secrete mostly IFN$\gamma$, while CD4+CD45RO+CXCR5+CXCR3-CCR6(cTfh2) cells secrete IL-4, IL-5, IL-13 and IL-21, and CD4+CD45RO+CXCR5+CXCR3-CCR6+ (cTfh17) cells secrete mainly IL-17, IL-21, and IL-22. Dysregulation of the cTfh cell compartment has been reported in autoimmune diseases, cancer and infections associated with abnormal B cell responses (Boswell et al., 2014; Ueno et al., 2015). However, whether Tfh cells contribute to the generation of the abnormal B cell responses observed during $T$. cruzi infection remains to be elucidated (Minoprio et al., 1988; Fernández et al., 2014). The aim of this work was to provide an insight in the adaptive immune response elicited by $T$. cruzi in humans. To this end, various peripheral blood CD4+CD45RO+CXCR5+ cell subsets were assessed in adult patients with different clinical forms of chronic Chagas disease. The results showed expansion of CCR6+ cTfh cells and decreased cTfh 2 cells in infected patients, regardless of the clinical status of the disease. Other phenotypic changes observed include expansion of cTfh 17 cells and decreased cTfh1 cells in asymptomatic patients -but not in patients with chagasic dilated cardiomyopathy-, which suggest that dysregulation of Tfh cells might contribute to Chagas disease progression.

\section{MATERIALS AND METHODS}

\section{Study Population}

A total of 31 adult participants were enrolled at the Clinical Facilities of Instituto Nacional de Parasitología "Dr. Mario Fatala Chabén," Buenos Aires. The clinical status of enrolled individuals was determined by physical examination and clinical tests, including serologic testing for T. cruzi infection, electrocardiography, chest radiography, and echocardiography. Individuals who had positive serology for T. cruzi were grouped according to the classification of Kuschnir et al. (1985) into: (a) patients with no clinical manifestations of heart disease, a normal electrocardiogram and conserved cardiac silhouette in chest X-rays and echocardiogram (ASYMP; Kuschnir group 0;n $=12$; mean age $\pm \mathrm{SD}=48.83 \pm 12.37$ years) and (b) patients with chronic Chagas dilated cardiomyopathy (CCC; Kuschnir group 2; $n=5$; mean age $\pm \mathrm{SD}=48.4 \pm 7.50$ ); clinically healthy individuals with a negative test for $T$. cruzi infection (CTRL, $n=14$; mean age $\pm \mathrm{SD}=41.71 \pm 13.36$ ) served as non-infected controls. Age of controls was matched as much as made possible to that of the infected individuals for each assay. A signed informed consent was obtained from all participants. All the research was conducted in accordance with the Declaration of Helsinki and the Council for International Organizations of Medical Sciences (CIOMS). The study protocol was approved by the Review Board of Instituto Nacional de Parasitología "Dr. Mario Fatala Chabén," Administración Nacional de Laboratorios e Institutos de Salud Dr. Carlos G. Malbrán (IRB00006651).

\section{Isolation of PBMC and Flow Cytometry Assays}

Freshly isolated peripheral blood mononuclear cells (PBMCs) were separated by density gradient centrifugation on FicollPaque Plus (GE Healthcare, Uppsala, Sweden) and stained with mAbs against CD4-FITC (Biolegend), CD45RO-PerCpCy5.5 (Biolegend), CXCR5-APC (eBioscience), CCR7-PE-Cy7 (BD Pharmingen), PD1-Pac Blue (Biolegend), CXCR3-PECy7 (Biolegend), CCR6-PE (Biolegend), CD19-PE-Cy7 (BD Pharmingen), IgD-PE (BD Pharmingen), and IgG-APC (BD Pharmingen), following the instructions of manufacturers. Cell 
viability was monitored in all samples by incorporating Fixable Viability Dye e-Fluor 780 (Invitrogen, Carlsbad, CA, USA) to the stains. At least 200,000 events per sample were collected at a FACSAria II flow cytometer (BD Immunocytometry Systems) and analyzed using FlowJo software (Tree Star, Inc., Ashland, OR, USA). Lymphocytes were identified by forward and sideangle light scatter characteristics. Non-specific fluorescence was defined with Fluorescence minus one (FMO) controls (Hulspas et al., 2009) (Supplementary Figure 1).

\section{Statistical Analysis}

Statistical analysis was performed using GraphPad Prism (GraphPad Software, Inc., La Jolla, CA, USA). The KolmogorovSmirnov normality distribution test was applied to all data. ANOVA followed by Bonferroni or Kruskal Wallis followed by Dunns were used for multiple comparisons. Comparisons between groups were made using unpaired Student's $t$-test or Mann-Whitney test. Pearson or Spearman tests were used to determine correlation between study parameters. A simple linear regression test was used for trend analysis. Values of $p<0.05$ were considered statistically significant.

\section{RESULTS}

\section{Identification of Circulating T Follicular Helper Cells in Trypanosoma cruzi-Infected Patients}

It has been well established that CXCR5 expression is essential for migration of $\mathrm{CD} 4+\mathrm{T}$ cells to $\mathrm{B}$ cell follicles, where they interact with cognate $\mathrm{B}$ cells and provide help for the generation of protective antibody responses (Breitfeld et al., 2000; Schaerli et al., 2000). We therefore asked whether chronic T. cruzi infection impacts on the circulating CD4+ cell population with homing capability to B cell follicles. To this end, we measured the expression of CXCR5 as frequency of overall circulating $\mathrm{CD} 4+$ cells in patients with different clinical forms of chronic Chagas disease and in healthy control subjects (Figure 1A). No statistically significant differences were found in the frequency of CXCR5+CD4+ cells among the study groups (Figure 1B). Since the CXCR5 marker has been shown to be expressed by recently activated naïve CD4+ cells and a subset of long-lived memory CD4+ cells (Schaerli et al., 2001; Nurieva et al., 2008; Schmitt et al., 2014), we next measured the expression of CD45RO on CXCR5+CD4+ cells. Most circulating CXCR5+CD4+ cells co-expressed the memory cell marker in our study population (Figure 1C), as described by other authors (Breitfeld et al., 2000; Schaerli et al., 2000; Schmitt et al., 2014). Further analysis of CD45RO expression on the overall circulating CD4+ cell population showed a significantly higher frequency of CD4+CD45RO+ cells in T. cruzi-infected subjects, as compared to control subjects, irrespective of the clinical status of Chagas heart disease ( $P=0.0041$; Figure 1D). Thus, cTfh cells were defined by the co-expression of CXCR5 and CD45RO within the CD4+ cell compartment (Figure 2A). The frequencies of CD4+CD45RO+CXCR5+ cells were not different among the study groups. However, the CXCR5 gMFI in ASYMP patients was significantly lower as compared to control subjects $(P=0.0438$; Figure 2B).

To determine whether T. cruzi infection leads to a change in the phenotype of cTfh cells, we next examined the expression of CCR7 (the chemokine receptor that controls homing to secondary lymphoid organs) and PD-1 (a molecule highly expressed by activated T cells) (Sallusto et al., 1999; Keir et al., 2008). CCC patients exhibited a statistically significant higher frequency of circulating CD4+CD45RO+CXCR5+CCR7+ cells, as compared to ASYMP patients ( $P=0.0048$; Figure 2C). On the other hand, most circulating CD4+CD45RO+CXCR5+ cells did not express PD-1, indicating that cTfh cells were in a resting status, irrespective of the infection and clinical condition (Figure 2D). Afterward, we evaluated the expression of chemokine receptors CXCR3 and CCR6 (classically linked to the production of IFN- $\gamma$ and IL-17, respectively; Singh et al., 2008; Groom and Luster, 2011) on CD4+CD45RO+CXCR5+ cells. No statistically significant differences were found in the frequency of CD4+CD45RO+CXCR5+CXCR3+ cells and CXCR3 gMFI levels among the study groups (Figure 2E). However, the frequencies of CD4+CD45RO+CXCR5+CCR6+ cells were significantly higher in both patient groups, as compared to controls $(P=0.0008$; Figure $2 \mathrm{~F})$. Besides, CCR6 gMFI on CD4+CD45RO+CXCR5+CCR6+ cells in ASYMP patients was significantly higher than that in control subjects $(P=0.0121)$. Noteworthy, the proportions of $\mathrm{CD} 4+\mathrm{CD} 45 \mathrm{RO}+\mathrm{CXCR} 5+\mathrm{CCR} 6+$ cells in ASYMP and CCC patients were significantly higher than those of CD4+CD45RO+CXCR5+CXCR3+ cells $(P=0.0001$ and $P=$ 0.0033 , respectively; Student's $t$-test), as opposed to the balanced distribution of these cells observed in healthy controls $(P=$ 0.6550 ; Student's $t$-test). Altogether, these data suggest that the infection with T. cruzi induces a shift of the cTfh compartment toward a Th17-like phenotype.

\section{Chronic Chagas Disease Is Associated With a Defective cTfh Cell Subset Composition}

The differential co-expression of CXCR3 and CCR6 was then used to define functionally distinct cTfh subsets (Figure 3A). ASYMP patients showed decreased cTfh1 cell frequencies, as compared to controls ( $P=0.0282$; Figure 3B); nevertheless, linear regression analysis revealed a negative trend in the frequency of these cells as the disease severity increased $(P=$ 0.0271, slope $=-3.747$ ). No differences in CXCR3 gMFI levels were observed among the study groups. The frequency of cTfh2 cells in ASYMP and CCC patients was significantly lower, as compared to controls $(P=0.0043$; Figure $3 \mathrm{C})$, with a negative trend in these cells as the infection was more severe $(P=$ 0.0026 , slope $=-3.747)$. Conversely, the frequency of cTfh 17 cells was significantly increased in ASYMP patients, as compared to controls $(P=0.0359$; Figure 3D). Additionally, CCR6 gMFI levels on these cells were significantly higher in ASYMP patients, as compared to both CCC patients and controls $(P=0.0178)$. The frequency of cTfh1/17 (Liu et al., 2017) cells was similar among the study groups (Figure $3 \mathbf{E}$ ), though a positive trend was 


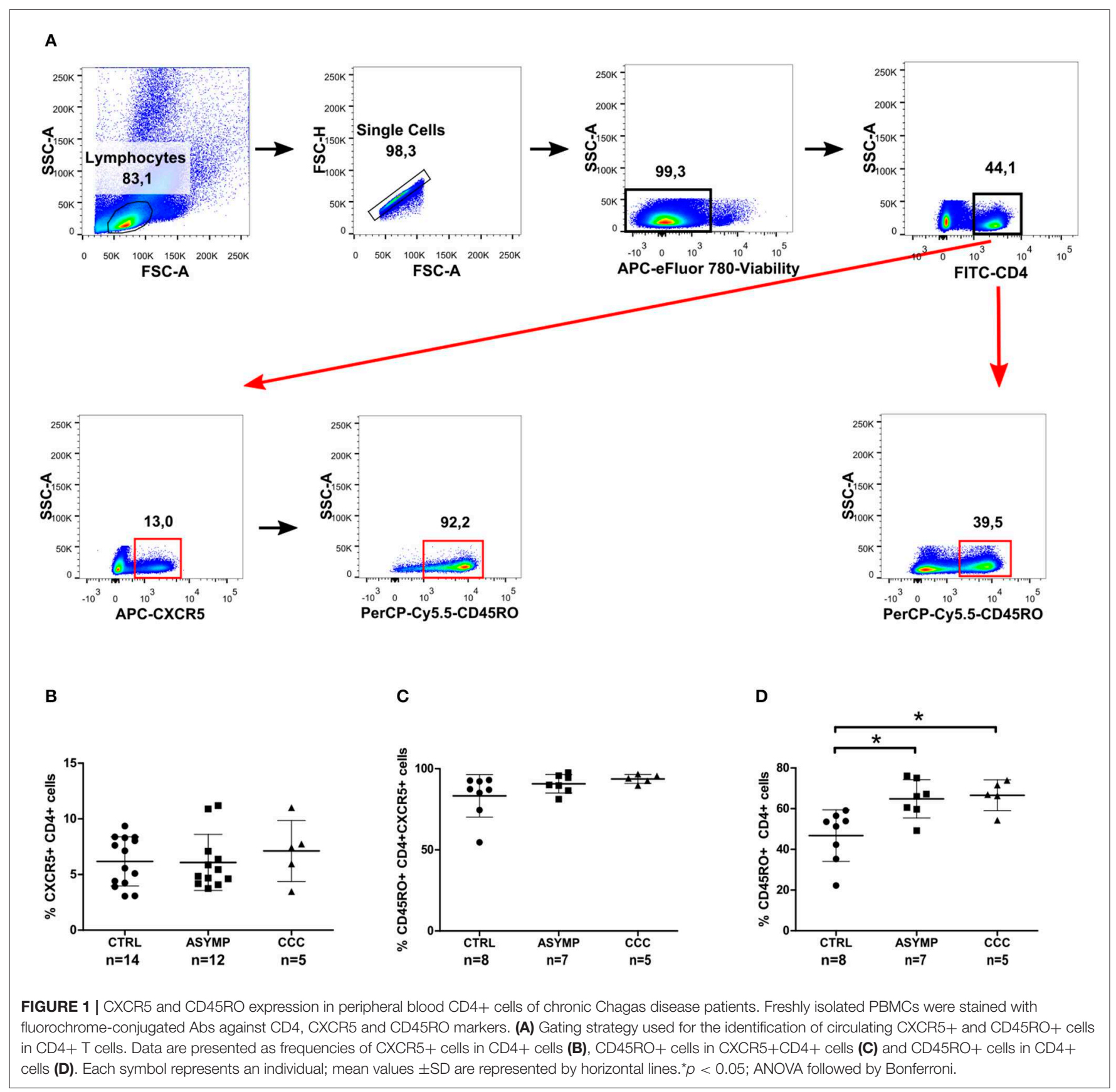

observed with disease progression ( $P=0.0327$, slope 3.921$)$. In these cells, CXCR3 gMFI levels were similar among the study groups; however, CCR6 gMFI levels were significantly higher in ASYMP -but not in CCC-patients, as compared to controls $(P=0.0182$; Figure 3E).

Previous studies from our lab have shown a progressive decrease in $T$. cruzi-specific CD4+ IFN- $\gamma+$ cell numbers as Chagas heart disease was more severe (Albareda et al., 2009). Other authors have reported that the Th1 effector cytokine IFN- $\gamma$ inhibits the development of Th17 effector responses (Harrington et al., 2005). T-bet, the transcription factor involved in the differentiation of Th1 cells has been demonstrated to inhibit $T$. cruzi-specific CD4+ Th17 cell responses in vivo (Guo et al., 2009). We here analyzed the relationship between different cTfh subsets by linear regression and found that the frequency of cTfh1 cells inversely correlated with that of cTfh17 cells in infected patients as well as in controls (Figure 4). No correlation between cTfh1 vs. cTfh2, and cTfh2 vs. cTfh 17 cell frequencies was found. Similarly, the frequency of $\mathrm{CD} 4+\mathrm{CD} 45 \mathrm{RO}+\mathrm{CXCR} 5+$ cells did not correlate with the frequency of circulating CD19+IgD-IgG+ B cells (Supplementary Table 1). We also failed to detect a relationship between the frequencies of cTfh subsets and serum levels 


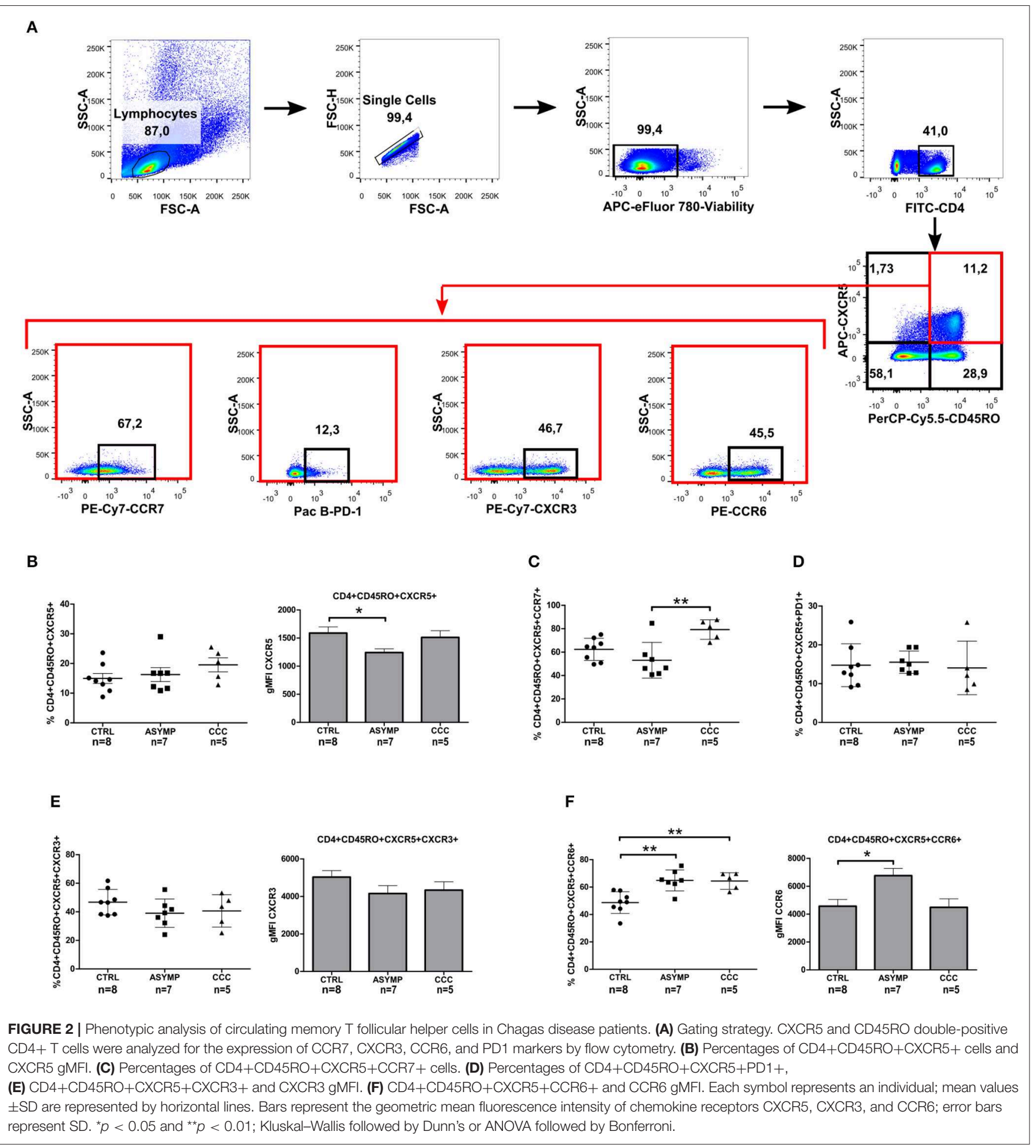

of T. cruzi-specific IgG antibodies, as measured by ELISA (data not shown).

As IFN- $\gamma$ and IL-17 were proposed to play critical roles in the pathogenesis of Chagas disease cardiomyopathy (Magalhães et al., 2013; Dutra et al., 2014), we extended our phenotypic analysis of CXCR3 and CCR6 expression to $\mathrm{CD} 4+$, CD4+CD45RO+ and CD4+CD45RO+CXCR5- (non-follicular memory) cells. No differences were observed in the frequencies of $\mathrm{CD} 4+$ cells and CD4+CD45RO+ cells expressing either marker among the study groups (Supplementary Table 1). The proportion of CXCR3+ cells within the CD4+CD45RO+CXCR5- compartment was significantly higher than that of CCR6+ cells in healthy controls $(P=0.0012$; Student $t$-test $)$, but not in infected 


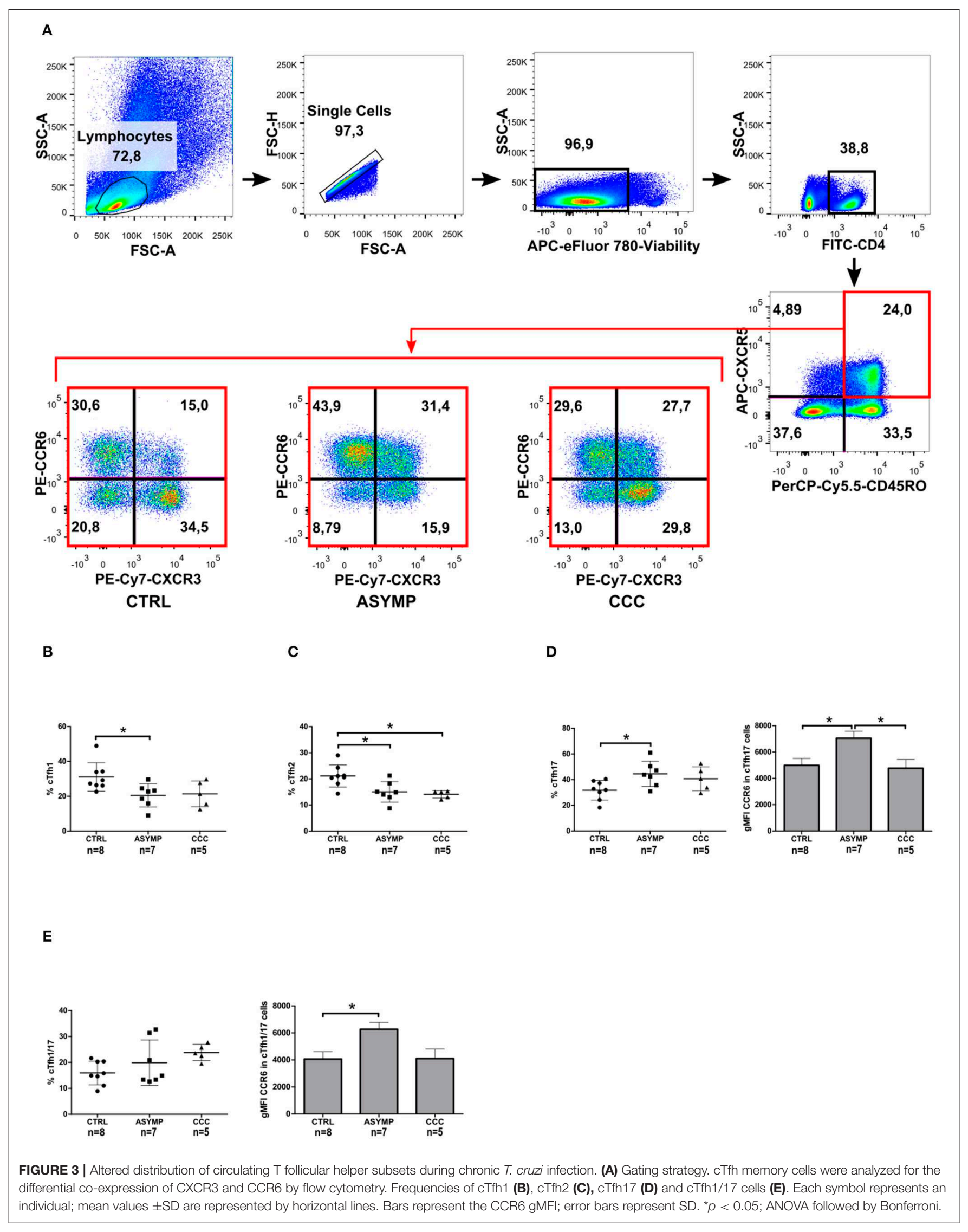




\section{CTRL}
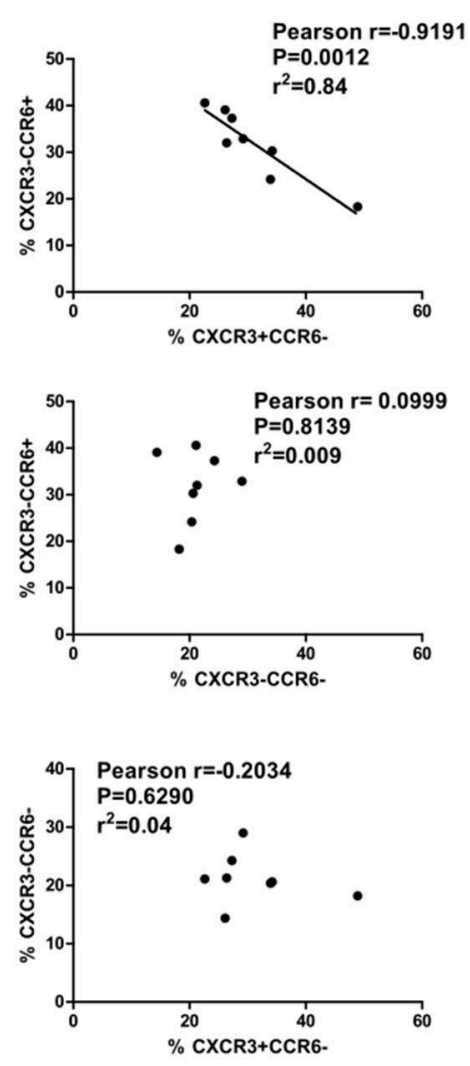

ASYMP
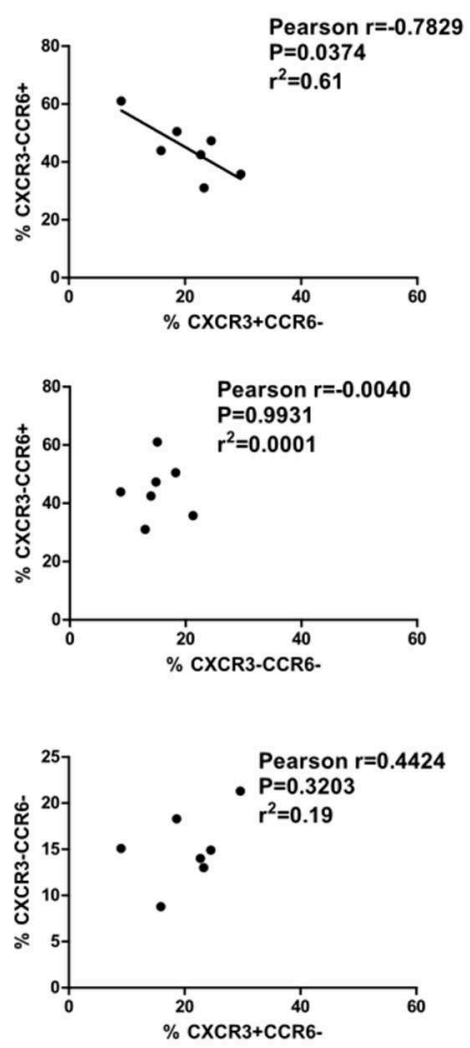

CCC
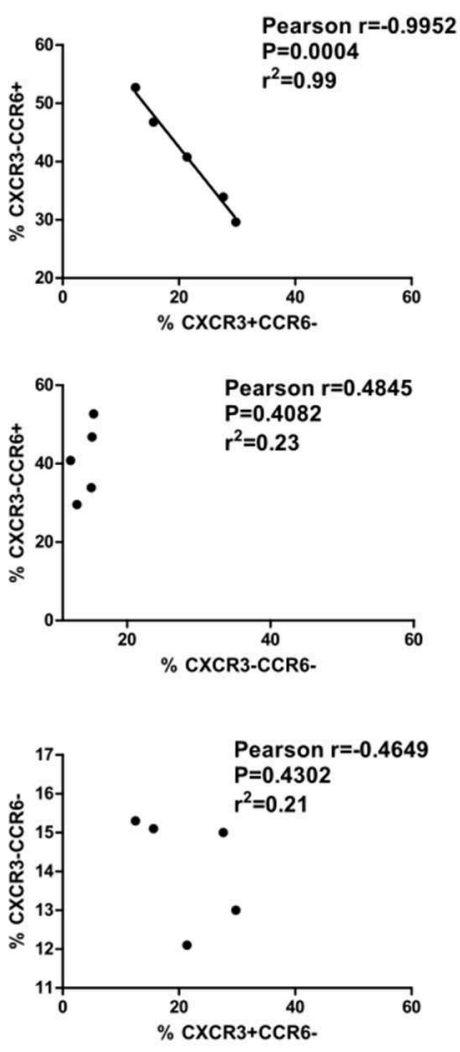

FIGURE 4 | Correlation analysis between cTfh subset frequencies. cTfh17 cell frequencies inversely correlate with cTfh1 cell frequencies in Chagas disease patients and healthy subjects. There is no correlation of cTfh 17 vs. cTfh2 cell frequencies, and cTfh1 vs. cTfh2 cell frequencies. $p<0.05$ was consider significant; Pearson correlation coefficient test.

patients $(p>0.05)$. These data suggest that Th1 polarization of non-follicular memory CD4+ cells does not occur in chronic Chagas disease patients. We next measured CXCR3 and CCR6 co-expression in CD4+CD45RO+CXCR5- cells and found similar frequencies of CXCR3+CCR6-, CXCR3-CCR6-, CXCR3-CCR6+ and CXCR3+CCR6+ cells among the study groups (Supplementary Table 1).

\section{DISCUSSION}

The relevance of abnormal $\mathrm{T}$ and $\mathrm{B}$ cell responses in the progression of chronic Chagas disease has received considerable attention. Multiple $\mathrm{T}$ cell subsets and cytokines seem to be involved in the pathogenesis of Chagas cardiomyopathy, but the exact mechanisms leading to parasite persistence and tissue damage have not been fully established. Classically, effector $\mathrm{T}$ helper cells include distinct subsets which can be distinguished by specific activating transcription factors, the profile of cytokines they secrete upon re-stimulation and the chemokines defining their migration properties (Deenick et al., 2011). T follicular helper cells represent a particular lineage of $\mathrm{CD} 4+$ helper cells that provide essential support to cognate
B cells for proliferation, somatic hypermutation, class-switch recombination, and selection of high affinity $B$ cells in germinal centers of secondary lymphoid organs (Crotty, 2015). In this study, we evaluated the expression of the chemokine receptors CXCR3 and CCR6 on CD4+CD45RO+CXCR5+ cells from patients chronically infected with $T$. cruzi. The results of our approach exposed a dysbalanced cTfh cell composition, which includes a progressive contraction of cTfh1 and cTfh2 cells, expansion of cTfh $1 / 17$ cells and increased frequency of CCR6+ cells as disease was more severe. Further analysis of cTfh 17 and cTfh1/17 exposed the association of increased CCR6 cell expression with asymptomatic disease, supporting the concept that IL-17 plays a protective role against the progression to Chagas cardiomyopathy proposed by other authors (Magalhães et al., 2013). The contraction of cTfh 1 cells was more marked in asymptomatic patients, and it was accompanied by the expansion of cTfh17 cells. It is plausible that the shift toward a Th17-like phenotype observed occurs during early stages of CD4+ T cell differentiation, since IFN- $\gamma$ and IL- 4 are known to antagonize naive, but not mature Th17 cells (Harrington et al., 2005). The exact mechanisms involved in the generation of expanded cTfh 17 cells and contracted cTfh 1 and cTfh 2 cells during chronic T. cruzi 
infection remains to be determined. This is the first report of the impact of chronic T. cruzi infection on the population of circulating Tfh cells. Although yet to be established, the altered composition of cTfh cells may reflect changes in germinal center dynamics that could play a role in the pathogenesis of Chagas disease. A better understanding of the functionality of these cells will likely provide new and important clues about the immune response against $T$. cruzi and the potential implication of cTfh cells in the progression to Chagas disease cardiomyopathy.

The differentiation of activated B cells into immunoglobulinsecreting cells in tonsils was demonstrated to be largely mediated by Tfh cell-derived IL-21 (Bryant et al., 2007). Different authors have demonstrated that peripheral blood Tfh17 and Tfh2 cells produce IL-21 more efficiently than other circulating Tfh and Th subpopulations, and are thus considered efficient B helper cells (Morita et al., 2011; Schmitt et al., 2014). In contrast with reports on humoral responses in autoimmune diseases (Morita et al., 2011), in this work we failed to demonstrate any association of cTfh subsets with the frequency of IgG+ mature B cells ex-vivo and the levels of serum T. cruzi-specific antibodies. This phenomenon could be attributed to humoral T. cruzispecific responses being regulated by mechanisms other than Tfh cells, including $\mathrm{T}$ cell-independent pathways, as proposed for human HIV infection (Boswell et al., 2014). Interestingly, massive extrafollicular B cell responses were described as an important source of non-specific antibodies in mice acutely infected with T. cruzi (Bermejo et al., 2011). Otherwise, peripheral blood Tfh cells in chronic T. cruzi infection might not fully represent the pool of Tfh cells residing in germinal centers.

Naïve CD4+ T cells are known to constitutively express CCR7, a key molecule for cell migration toward CCL19 and CCL21 ligands expressed in the T-zones of secondary lymphoid organs. Diverse cytokines and inflammatory mediators have been shown to modulate the expression of CCR7 and immune cell activation (Comerford et al., 2013). The attenuation of CCR7 expression and the induction of CXCR5 facilitate the entry of CD4+ cells into B cell follicles and GC in response to CXCL13 expression; CCR7 recycles back to the cell membrane after egressing from lymph nodes in a sphingosine-1-phosphate receptor-1 (S1P1)- dependent manner (Matloubian et al., 2004). Conversely, the maintenance of CCR7 expression was shown to inhibit the access of CXCR5+ cells to follicles (Haynes et al., 2007). Chronic chagasic cardiomyopathy is characterized by myocardial cell necrosis, fibrosis and active inflammatory process involving CD8+ and CD4+ T cells, NK cells and macrophages which secrete mainly proinflamatory cytokines (Dutra et al., 2014). It is possible that inflammatory mediators associated with cardiac lesions induce the expression of CCR7 on CXCR5 + cells, promoting the re-circulation of CCR7+ cTfh cells and their accumulation in the peripheral blood.

An additional finding of our study was the expansion of peripheral blood CD4+CD45RO+ cells associated with chronic T. cruzi infection, which contrasts with the unaltered proportion of CD4+CD45RO+ cells ex-vivo reported by Fiuza et al. (2009). It is possible that this discrepancy be due to the influence of parasite and host genetic diversity on the outcome of human Chagas disease, as suggested by other authors (Santos D et al., 2009; Zingales et al., 2012; Poveda et al., 2014).
In summary, in this work we characterized circulating Tfh cells in patients chronically infected with T. cruzi and demonstrated an imbalance in the distribution of cTfh subsets associated with the clinical forms of Chagas disease. The major weakness of the present study is the relatively low number of patients with dilated chagasic cardiomyopathy included in the analysis. Moreover, we were unable to obtain data on the cytokine profile and functionality of cTfh cells that could lead to a better understanding of their role in the pathogenesis of chronic Chagas heart disease. However, our detailed phenotypic characterization provides indirect evidence of a potential role of Tfh cells in the abnormal immune responses against T. cruzi (Fernández et al., 2014), establishing the basis to perform further studies to assess these processes.

\section{DATA AVAILABILITY STATEMENT}

The datasets generated for this study are available on request to the corresponding author.

\section{ETHICS STATEMENT}

The studies involving human participants were reviewed and approved by the Review Board of Instituto Nacional de Parasitología Dr. Mario Fatala Chabén, Administración Nacional de Laboratorios e Institutos de Salud Dr. Carlos G. Malbrán (IRB00006651). The patients/participants provided their written informed consent to participate in this study.

\section{AUTHOR CONTRIBUTIONS}

MP conceived the project and designed research. MP and LQ performed the experiments, interpreted data, and wrote the paper. $\mathrm{YH}-\mathrm{V}$ enrolled and performed clinical evaluation of participants. EF, PP, and $\mathrm{YH}-\mathrm{V}$ interpreted data, validated, and edited the paper. MP and PP acquired funding.

\section{FUNDING}

This work was partially supported by grant of Agencia Nacional de Promoción Científica y Tecnológica, Fondo para la Investigación Científica y Tecnológica FONCyT PICT 2013-2828 to MP.

\section{ACKNOWLEDGMENTS}

We thank all the volunteers who provided blood samples for the study. We highly appreciate the skillful technical assistance of Mrs. Daniela Oliveto along with the Staff at the Diagnostic Department of the INP.

\section{SUPPLEMENTARY MATERIAL}

The Supplementary Material for this article can be found online at: https://www.frontiersin.org/articles/10.3389/fcimb. 2020.00126/full\#supplementary-material 


\section{REFERENCES}

Acosta-Rodriguez E, V., Rivino, L., Geginat, J., Jarrossay, D., Gattorno, M., Lanzavecchia, A., et al. (2007). Surface phenotype and antigenic specificity of human interleukin 17-producing T helper memory cells. Nat. Immunol. 8, 639-646. doi: 10.1038/ni1467

Albareda, M. C., Olivera, G. C., Laucella, S. A., Alvarez, M. G., Fernandez, E. R., Lococo, B., et al. (2009). Chronic human infection with Trypanosoma cruzi drives CD4+ T cells to immune senescence. J. Immunol. 183, 4103-4108. doi: 10.4049/jimmunol.0900852

Bermejo, D. A., Amezcua Vesely, M. C., Khan, M., Acosta Rodríguez E, V., Montes, C. L., Merino, M. C., et al. (2011). Trypanosoma cruzi infection induces a massive extrafollicular and follicular splenic B-cell response which is a high source of non-parasite-specific antibodies. Immunology 132, 123-133. doi: 10.1111/j.1365-2567.2010.03347.x

Boswell, K. L., Paris, R., Boritz, E., Ambrozak, D., Yamamoto, T., Darko, S., et al. (2014). Loss of circulating $\mathrm{CD} 4 \mathrm{~T}$ cells with $\mathrm{B}$ cell helper function during chronic HIV infection. PLoS Pathog. 10:e1003853. doi: 10.1371/journal.ppat.1003853

Breitfeld, D., Ohl, L., Kremmer, E., Ellwart, J., Sallusto, F., Lipp, M., et al. (2000). Follicular B helper T cells express CXC chemokine receptor 5, localize to B cell follicles, and support immunoglobulin production. J. Exp. Med. 192, 1545-1552. doi: 10.1084/jem.192.11.1545

Bryan, M. A., Guyach, S. E., and Norris, K. A. (2010). Specific humoral immunity versus polyclonal B cell activation in Trypanosoma cruzi infection of susceptible and resistant mice. PLoS Negl. Trop. Dis. 4:e733. doi: 10.1371/journal.pntd.0000733

Bryant, V. L., Ma, C. S., Avery, D. T., Li, Y., Good, K. L., Corcoran, L. M., et al. (2007). Cytokine-mediated regulation of human B cell differentiation into Igsecreting cells: predominant role of IL-21 produced by CXCR5+ T follicular helper cells. J. Immunol. 179, 8180-8190. doi: 10.4049/jimmunol.179.12.8180

Cardoso, M. S., Reis-Cunha, J. L., and Bartholomeu, D. C. (2016). Evasion of the immune response by Trypanosoma cruzi during acute infection. Front. Immunol. 6:659. doi: 10.3389/fimmu.2015.00659

Comerford, I., Harata-Lee, Y., Bunting, M. D., Gregor, C., Kara, E. E., and McColl, S. R. (2013). A myriad of functions and complex regulation of the CCR7/CCL19/CCL21 chemokine axis in the adaptive immune system. Cytokine Growth Factor Rev. 24, 269-283. doi: 10.1016/j.cytogfr.2013.03.001

Crotty, S. A. (2015). brief history of T cell help to B cells. Nat. Rev. Immunol. 15, 185-189. doi: 10.1038/nri3803

Cunha-Neto, E., Teixeira, P. C., Nogueira, L. G., and Kalil, J. (2011). Autoimmunity. Adv. Parasitol. 76, 129-152. doi: 10.1016/B978-0-12-385895-5.00006-2

Deenick, E. K., Ma, C. S., Brink, R., and Tangye, S. G. (2011). Regulation of T follicular helper cell formation and function by antigen presenting cells. Curr. Opin. Immunol. 23, 111-118. doi: 10.1016/j.coi.2010.10.007

Dutra, W. O., Menezes, C. A. S., Magalhães, L. M. D., and Gollob, K. J. (2014). Immunoregulatory networks in human Chagas disease. Parasite Immunol. 36, 377-387. doi: 10.1111/pim.12107

Fernández, E. R., Olivera, G. C., Quebrada Palacio, L. P., González, M. N., Hernandez-Vasquez, Y., Sirena, N. M., et al. (2014). Altered distribution of peripheral blood memory B cells in humans chronically infected with Trypanosoma cruzi. PLoS ONE 9:e104951. doi: 10.1371/journal.pone.0104951

Fiuza, J. A., Fujiwara, R. T., Gomes, J. A., Rocha, M. O., Chaves, A. T., de Araújo, F. F., Fares, R. C., et al. (2009). Profile of central and effector memory T cells in the progression of chronic human chagas disease. PLoS Negl. Trop. Dis. 3:e512. doi: 10.1371/journal.pntd.0000512

Gomes, J. A., Bahia-Oliveira, L. M., Rocha, M. O., Martins-Filho, O. A., Gazzinelli, G., and Correa-Oliveira, R. (2003). Evidence that development of severe cardiomyopathy in human Chagas' disease is due to a Th1-specific immune response. Infect. Immun. 71, 1185-1193. doi: 10.1128/iai.71.3.1185-1193.2003

Groom, J. R., and Luster, A. D. (2011). CXCR3 in T cell function. Exp. Cell. Res. 317, 620-631. doi: 10.1016/j.yexcr.2010.12.017

Guo, S., Cobb, D., and Smeltz, R. B. (2009). T-bet inhibits the in vivo differentiation of parasite-specific CD4+ Th17 cells in a T cell-intrinsic manner. J. Immunol. 182, 6179-6186. doi: 10.4049/jimmunol.0803821

Harrington, L. E., Hatton, R. D., Mangan, P. R., Turner, H., Murphy, T. L., Murphy, K. M., et al. (2005). Interleukin 17-producing CD4+ effector T cells develop via a lineage distinct from the T helper type 1 and 2 lineages. Nat. Immunol. 6 , 1123-1132. doi: 10.1038/ni1254

Haynes, N. M., Allen, C. D. C., Lesley, R., Ansel, K. M., Killeen, N., and Cyster, J. G. (2007). Role of CXCR5 and CCR7 in follicular Th cell positioning and appearance of a programmed cell death gene-1high germinal center-associated subpopulation. J. Immunol. 179, 5099-5108. doi: 10.4049/jimmunol.179.8.5099

Hulspas, R., O'Gorman, M. R. G., Wood, B. L., Gratama, J. W., and Sutherland, D. R. (2009). Considerations for the control of background fluorescence in clinical flow cytometry. Cytometry B Clin. Cytom. 76, 355-364. doi: 10.1002/cyto.b.20485

Keir, M. E., Butte, M. J., Freeman, G. J., and Sharpe, A. H. (2008). PD-1 and its ligands in tolerance and immunity. Annu. Rev. Immunol. 26, 677-704. doi: 10.1146/annurev.immunol.26.021607.090331

Kuschnir, E., Sgammini, H., Castro, R., Evequoz, C., Ledesma, R., and Brunetto, J. (1985). [Evaluation of cardiac function by radioisotopic angiography, in patients with chronic Chagas cardiopathy]. Arq. Bras. Cardiol. 45, 249-256.

Liu, D., Liu, J., Wang, J., Guo, L., Liu, C., Jiang, Y., et al. (2017). Distribution of circulating $\mathrm{T}$ follicular helper cell subsets is altered in immunoglobulin A vasculitis in children. PLoS ONE 12:e0189133. doi: 10.1371/journal.pone.0189133

Magalhães, L. M., Villani, F. N., Nunes M do, C., Gollob, K. J., and Rocha, M. O., Dutra, W. O. (2013). High interleukin 17 expression is correlated with better cardiac function in human Chagas disease. J. Infect. Dis. 207, 661-665. doi: 10.1093/infdis/jis724

Matloubian, M., Lo, C. G., Cinamon, G., Lesneski, M. J., Xu, Y., Brinkmann, V., et al. (2004). Lymphocyte egress from thymus and peripheral lymphoid organs is dependent on S1P receptor 1. Nature 427, 355-360. doi: 10.1038/ nature 02284

Minoprio, P., Burlen, O., Pereira, P., Guilbert, B., Andrade, L., HontebeyrieJoskowicz, M., et al. (1988). Most B cells in acute Trypanosoma cruzi infection lack parasite specificity. Scand. J. Immunol. 28, 553-561. doi: 10.1111/j.1365-3083.1988.tb01487.x

Morita, R., Schmitt, N., Bentebibel, S., Ranganathan, R., Bourdery, L., Zurawski, G., et al. (2011). Human blood CXCR5(+)CD4(+) T cells are counterparts of T follicular cells and contain specific subsets that differentially support antibody secretion. Immunity 34, 108-121. doi: 10.1016/j.immuni.2010.12.012

Nurieva, R. I., Chung, Y., Hwang, D., Yang, X. O., Kang, H. S., Ma, L., et al. (2008). Generation of $\mathrm{T}$ follicular helper cells is mediated by interleukin-21 but independent of T helper 1, 2, or 17 cell lineages. Immunity 29, 138-149. doi: 10.1016/j.immuni.2008.05.009

Poveda, C., Fresno, M., Gironès, N., Martins-Filho, O. A., Ramírez, J. D., Santi-Rocca, J., et al. (2014). Cytokine profiling in chagas disease: towards understanding the association with infecting Trypanosoma cruzi discrete typing units (a BENEFIT TRIAL sub-study). PLoS ONE 9:e91154. doi: 10.1371/journal.pone.0091154

Quebrada Palacio, L. P., González, M. N., Hernandez-Vasquez, Y., Perrone, A. E., Parodi-Talice, A., Bua, J., et al. (2018). Phenotypic diversity and drug susceptibility of Trypanosoma cruzi TcV clinical isolates. PLoS ONE 13:203462. doi: 10.1371/journal.pone.0203462

Sallusto, F., Lenig, D., Förster, R., and Lipp, M., Lanzavecchia, A. (1999). Two subsets of memory $\mathrm{T}$ lymphocytes with distinct homing potentials and effector functions. Nature 401, 708-712. doi: 10.1038/44385

Santos D, d, Talvani, A., da Mata Guedes, P. M., Machado-Coelho, G. L. L., de Lana, M., and Bahia, M. T. (2009). Trypanosoma cruzi: Genetic diversity influences the profile of immunoglobulins during experimental infection. Exp. Parasitol. 121, 8-14. doi: 10.1016/j.exppara.2008.09.012

Schaerli, P., Loetscher, P., and Moser, B. (2001). Cutting edge: induction of follicular homing precedes effector Th cell development. J. Immunol. 167, 6082-6086. doi: 10.4049/jimmunol.167.11.6082

Schaerli, P., Willimann, K., Lang, A. B., Lipp, M., Loetscher, P., and Moser, B. (2000). CXC chemokine receptor 5 expression defines follicular homing T cells with B cell helper function. J. Exp. Med. 192, 1553-1562. doi: $10.1084 /$ jem.192.11.1553

Schmitt, N., Bentebibel, S.-E., and Ueno, H. (2014). Phenotype and functions of memory Tfh cells in human blood. Trends Immunol. 35, 436-442. doi: 10.1016/j.it.2014.06.002

Singh, S. P., Zhang, H. H., Foley, J. F., Hedrick, M. N., and Farber, J. M. (2008). Human $\mathrm{T}$ cells that are able to produce IL-17 express the 
chemokine receptor CCR6. J. Immunol. 180, 214-221. doi: 10.4049/jimmunol. 180.1.214

Ueno, H., Banchereau, J., and Vinuesa, C. G. (2015). Pathophysiology of T follicular helper cells in humans and mice. Nat. Immunol. 16, 142-152. doi: 10.1038/ni.3054

Vitelli-Avelar, D. M., Sathler-Avelar, R., Teixeira-Carvalho, A., Pinto Dias, J. C., Gontijo, E. D., Faria, A. M., et al. (2008). Strategy to assess the overall cytokine profile of circulating leukocytes and its association with distinct clinical forms of human Chagas disease. Scand J. Immunol. 68, 516-525. doi: 10.1111/j.1365-3083.2008.02167.x

Watanabe Costa, R., da Silveira, J. F., and Bahia, D. (2016). Interactions between Trypanosoma cruzi secreted proteins and host cell signaling pathways. Front. Microbiol. 7:388. doi: 10.3389/fmicb.2016.00388

WHO World Health Organization (2018). Chagas disease (American trypanosomiasis), Fact Sheet. Available online at: https://www.who.int/ chagas/en/ (accessed October 21, 2019).
Zingales, B., Miles, M. A., Campbell, D. A., Tibayrenc, M., Macedo, A. M., Teixeira, M. M. G., et al. (2012). The revised Trypanosoma cruzi subspecific nomenclature: Rationale, epidemiological relevance and research applications. Infect. Genet. Evol. 12, 240-253. doi: 10.1016/j.meegid.2011.12.009

Conflict of Interest: The authors declare that the research was conducted in the absence of any commercial or financial relationships that could be construed as a potential conflict of interest.

Copyright (c) 2020 Quebrada Palacio, Fernández, Hernández-Vásquez, Petray and Postan. This is an open-access article distributed under the terms of the Creative Commons Attribution License (CC BY). The use, distribution or reproduction in other forums is permitted, provided the original author(s) and the copyright owner(s) are credited and that the original publication in this journal is cited, in accordance with accepted academic practice. No use, distribution or reproduction is permitted which does not comply with these terms. 